\title{
Evaluation of the appropriateness of imipenem/cilastatin prescription and dosing in a tertiary care hospital
}

This article was published in the following Dove Press journal:

Infection and Drug Resistance

24 March 2015

Number of times this article has been viewed

\section{Wissam K Kabbara \\ George T Nawas \\ Wijdan H Ramadan}

Department of Pharmacy Practice, School of Pharmacy, Lebanese American University, Byblos, Lebanon
Background: Imipenem/cilastatin is an antibacterial agent of the carbapenem class of $\beta$-lactams that is known to have an extremely wide spectrum of activity against Gram-positive, Gramnegative, aerobic, anaerobic, and even multidrug-resistant strains. The objective of this study was to evaluate the appropriate use of imipenem/cilastatin in a local tertiary care hospital. The study assessed the indication both empirically and after the culture results were available, the dose and dose adjustment in renal failure, as well as the incidence of seizure in hospitalized patients receiving imipenem/cilastatin.

Methods: This observational study was conducted in a tertiary care hospital over a 3-month period. The treatment of 100 patients with imipenem/cilastatin was evaluated both empirically and after culture results were available. Analysis of the appropriateness of imipenem/cilastatin indication, dose, and monitoring of seizure frequency was based on the package insert, updated published guidelines, and clinical judgment.

Results: Patients from internal medicine and intensive care units comprised approximately $50 \%$ of the population in the study. The patients received imipenem/cilastatin mainly for urinary tract infections (27\%) or for sepsis of an unknown focus (22\%). The use of imipenem/cilastatin empirically was appropriate in $97.2 \%(n=69 / 71)$ of the cases, and its use postculture in $86 \%$ of the cases. There were $29 \%$ of the patients who were not started on imipenem/cilastatin empirically. Four patients out of the 29 patients $(13.8 \%)$ who were not started on imipenem/cilastatin empirically inappropriately received imipenem/cilastatin post-culture results. Thirty-three patients $(33 \%)$ were not dosed appropriately, 30 of whom had renal impairment and creatinine clearance fluctuations. Only one patient developed a seizure while on imipenem/cilastatin.

Conclusion: The prescription of imipenem/cilastatin at our setting was mostly appropriate to what is recommended in the guidelines and the literature, although a few cases could have been managed better. Dosage adjustment, however, was not as appropriate, mainly in patients who did not have a stable creatinine clearance.

Keywords: imipenem, cilastatin, indication, dose, seizure

\section{Introduction}

Antimicrobial resistance is recognized as one of the greatest threats to human health worldwide. ${ }^{1}$ Before the widespread use of antibiotics, there were low levels of pre-existing antibiotic-resistant bacteria. ${ }^{2}$ Evolutionary pressure requiring the recurrent use of antibiotics directly contributed to the development of multidrugresistant microorganisms and the spread of resistance among bacterial species. ${ }^{3}$ The demand for the increased use of antibiotics as well as noncompliance with infectious disease guidelines both contribute to the rise of bacterial resistance. ${ }^{4}$
Correspondence: Wijdan H Ramadan Department of Pharmacy Practice, School of Pharmacy, Lebanese American University, PO Box 36-F53, Byblos, Lebanon

Tel +96I 9547249 ext 2267

Fax +96I 9547256 ext 2897

Emailwramadan@lau.edu.lb 
Seven decades of medical advances enabled by antibiotics are now threatened by the rising antibiotic resistance and the withdrawal of most major pharmaceutical companies from the antibiotics market. ${ }^{5}$ Among infections caused by Gram-positive pathogens, methicillin-resistant Staphylococcus aureus has taken center stage, now accounting for well over $50 \%$ of all documented staphylococcal infections in the US. ${ }^{6}$ In addition, Gram-negative bacteria, are also showing a decreased susceptibility to existing antibiotics. Most alarming is the evolution of extended-spectrum $\beta$-lactamase-producing Enterobacteriaceae, as well as the multidrug-resistant Pseudomonas aeruginosa (some strains are even resistant to carbapenems). P. aeruginosa in the US is reported to have $20 \%$ resistance rates to imipenem/ cilastatin, a drug that is frequently utilized when a suspicion of an infection by that organism is high. ${ }^{7,8}$ Similarly, in Lebanon, the resistance rate of $P$. aeruginosa to imipenem is approximately $20 \%{ }^{9}$

Resistance rates have increased among several other Gram-negative pathogens that are often responsible for serious nosocomial infections, including Acinetobacter species and other Enterobacteriaceae. ${ }^{10}$ The presence of multidrugresistant strains of these organisms has been associated with very bad outcomes, including prolonged hospital stays, higher health care costs, and increased mortality, especially when the initial antibiotic therapy does not provide adequate coverage of the causative pathogen. ${ }^{10}$ In 2004, the Centers for Disease Control and Prevention reported an increasing number of multidrug-resistant Acinetobacter baumannii bloodstream infections. ${ }^{11}$

Multidrug-resistant strains of Acinetobacter species are being isolated with increasing frequency in many of the nosocomial infections, especially nosocomial pneumonia, bloodstream infections, surgical site infections, and urinary tract infections. ${ }^{10}$ P. aeruginosa is also responsible for a wide range of severe nosocomial infections, including pneumonia, urinary tract infections, and bacteremia. ${ }^{12}$ In addition to its intrinsic resistance due to its rigid cell well, $P$. aeruginosa has also acquired resistance via multiple mechanisms, including production of $\beta$-lactamases and carbapenemases. ${ }^{13}$ Data from the National Nosocomial Infections Surveillance system in the US indicate that the incidence of nosocomial infections caused by Acinetobacter species and by P. aeruginosa increased drastically between 1975 and 2003. ${ }^{14}$

Imipenem/cilastatin, the very first semisynthetic thienamycin, is an antibacterial agent of the carbapenem class of $\beta$-lactams that is known to have an extremely wide spectrum of antibacterial activity against Gram-positive, Gram-negative, aerobic, anaerobic, and even multidrug-resistant strains. ${ }^{15}$ It is active against a wide variety of aerobic and anaerobic bacteria that are resistant to other agents, including resistant strains of S. aureus, enterococci, Enterobacteriaceae, P. aeruginosa, and Bacteroides fragilis.

Extensive clinical research marked the effectiveness of an initial monotherapy of imipenem/cilastatin in the treatment of moderate to severe infections, including nosocomial pneumonia, septicemia, febrile neutropenia, and intra-abdominal, diabetic foot, urinary tract, skin and soft tissue, as well as bone and joint infections. ${ }^{16}$ The dose of imipenem should be based on the location and severity of the infection, the susceptibility of the infecting pathogen(s), and the renal function of the patient. Adult patients with impaired renal function, as judged by creatinine clearance $(\mathrm{CrCl})<70 \mathrm{~mL} / \mathrm{min} / 1.73 \mathrm{~m}^{2}$, require dosage adjustment.

Many studies have addressed the appropriateness of imipenem/cilastatin prescription and dosing in health care institutions. Shiva et al, ${ }^{17}$ for instance, evaluated the utilization of imipenem/cilastatin in an educational hospital in Iran and found that there was a high empirical prescription of imipenem/cilastatin without considering culture and antimicrobial susceptibility results, and they found out that there was a lack of attention to dosage adjustments in patients with renal insufficiency. In another drug utilization evaluation conducted by Sakhaiyan et al, ${ }^{18}$ it was shown that dosage adjustment of imipenem/cilastatin was not being conducted appropriately at their institution, and the authors concluded that there was a need to further educate the health care professionals since the adjustment is dependent on the weight and the renal function of the patient.

Seizure occurrence with the use of imipenem/cilastatin has also been addressed extensively before. It was first reported by the manufacturer to be $0.4 \%$. However, subsequent postmarketing surveys demonstrated that imipenem/cilastatin can be associated with seizures more frequently. ${ }^{19-21}$ Calandra et al ${ }^{22}$ reviewed 1,754 patients treated with imipenem/cilastatin to determine the seizure risk associated with carbapenems. Patients were moderately to severely ill, with numerous risk factors for seizures. Seizures occurred in $3 \%$ of patients, but only $0.9 \%$ of them were judged to be possibly, probably, or definitely related to imipenem/cilastatin. As with other $\beta$-lactam antibiotics, central nervous system (CNS) disorders and renal insufficiency were risk factors for seizure occurrence. The patients who received imipenem/cilastatin at higher than recommended doses had an increased risk of 
seizures, especially the subgroup of patients with renal insufficiency. ${ }^{22}$

The objective of this study is to evaluate the appropriateness of imipenem/cilastatin prescription and dosing in a local tertiary care hospital. The study assesses the indication both empirically and after the culture results are available, the dose and the dose adjustment in renal failure, as well as the occurrence of seizures in patients on imipenem/cilastatin.

\section{Methods}

\section{Setting and design}

This study was designed to be of a prospective, observational nature. However, in order to have an acceptable sample size $(\mathrm{n}=100$ patients), a retrospective patient evaluation was also included. This observational study was conducted in a tertiary 544-bed teaching hospital over a 3-month period. Information on 100 patients receiving imipenem/cilastatin for treatment of infections in all hospital units was collected using a data collection form that included the following: demographic data, allergy history, past medical history, history of present illness, history of CNS diseases and/or seizure, site(s) of infection, imipenem/cilastatin dosage, frequency of administration, bacterial culture(s) and sensitivity results, temperature, white blood cell count, neutrophil count, serum creatinine, and calculated $\mathrm{CrCl}$ according to the Cockcroft-Gault equation in adults and the Schwartz equation in pediatrics. A few patients did not have their weights documented, so their weight was estimated and then used in the calculation of their estimated $\mathrm{CrCl}$. Electronic medical charts/records on all patients receiving imipenem/ cilastatin were also reviewed. The study was approved by the Institutional Review Board, and a waiver of consent was obtained to fill out the data collection form.

Imipenem/cilastatin treatment was evaluated both empirically and after the culture results were out. The analysis included the data collected from the initiation of the first dose of imipenem/cilastatin until patient discharge and/or discontinuation of the antibiotic. Analysis of the appropriateness of the use of imipenem/cilastatin was evaluated based on the Infectious Diseases Society of America guidelines, ${ }^{23-27}$ the Surviving Sepsis Campaign guidelines, ${ }^{28}$ drug information handbook dosage recommendations, ${ }^{29-31}$ and clinical judgment. The data collection form and final project report did not include any subject identifiers.

After obtaining the culture results, imipenem/cilastatin was considered to be appropriate if it was the only antibiotic to which the microorganism was sensitive to and if de-escalation to a narrower spectrum antibiotic was not possible.

\section{Sources of data}

An electronic daily list of all patients prescribed imipenem/ cilastatin was provided by the central pharmacy. Patients who received imipenem/cilastatin for treatment were then identified using the hospital's computerized chart system. Indication, dose, and clinical improvement or worsening of the symptoms of patients receiving imipenem/cilastatin were followed until discontinuation of the antibiotic. Bacterial cultures were also followed up throughout the duration of the treatment.

\section{Statistical analysis}

Data were processed and analyzed through the application of the software Statistical Package for Social Sciences (SPSS, version 19, IBM Corporation, Armonk, NY). Responses were tabulated and cross-tabulated, and percentages were calculated.

\section{Results \\ Patient distribution}

Over the 3-month period of the study, the electronic medical charts/records of 100 patients receiving imipenem/cilastatin were reviewed. The study patients were distributed over many hospital units, with the largest proportions from internal medicine $(28 \%)$ and intensive care $(21 \%)$ units. The others were distributed over the surgery (16\%), oncology $(13 \%)$, pediatrics $(12 \%)$, and cardiac care $(10 \%)$ units (Table 1).

Table I Demographic and clinical characteristics of the patients $(n=100)$

\begin{tabular}{ll}
\hline Sex & \\
Male & $59(59 \%)$ \\
Female & $41(41 \%)$ \\
Age ${ }^{a}$ (years) & $54.9 \pm 25.6$ \\
Diagnosis & \\
Urinary tract infection & $27(27 \%)$ \\
Sepsis (unknown focus) & $22(22 \%)$ \\
Nosocomial pneumonia & $19(19 \%)$ \\
Febrile neutropenia & $10(10 \%)$ \\
Unknown focus & $9(9 \%)$ \\
Skin and soft tissue infection & $4(4 \%)$ \\
Diabetic foot infection & $2(2 \%)$ \\
Intra-abdominal infection & $2(2 \%)$ \\
Bone and joint infection & $2(2 \%)$ \\
Others & $3(3 \%)$ \\
Wards & \\
Internal medicine & $28(28 \%)$ \\
Intensive care unit & $21(21 \%)$ \\
Surgery & $16(16 \%)$ \\
Oncology & $13(13 \%)$ \\
Pediatrics & $12(12 \%)$ \\
Cardiac care unit & $10(10 \%)$ \\
\hline
\end{tabular}

Notes: aValues are presented as mean \pm standard deviation. Median: 61.5 years; interquartile range: 33.7 years. 
A summary of the demographics of the patients in this study is shown in Table 1. The majority of patients on imipenem/cilastatin were males (59\%). The age of the patients ranged from 1 day to 90 years with a standard deviation of 25.6 years. The patients' baseline estimated $\mathrm{CrCl}$ ranged from $9 \mathrm{~mL} / \mathrm{min}$ to $255 \mathrm{~mL} / \mathrm{min}$ with a standard deviation of $83.61 \mathrm{~mL} / \mathrm{min}$. The standard deviation values are considerably high for both the age and the $\mathrm{CrCl}$ of the patients, which proves the wide variability of those two particular data sets among patients.

According to the guidelines, the patients were classified in groups according to their $\mathrm{CrCl}$. The average $\mathrm{CrCl}$ was calculated for every patient while at the hospital, according to the Cockcroft-Gault equation, and every patient had their serum creatinine regularly monitored for any requirement of dose adjustment, including hemodialysis patients. Fortynine percent (49\%) of the patients had a $\mathrm{CrCl} \geq 70 \mathrm{~mL} / \mathrm{min}$ and required no dosage adjustment. The remaining patients (51\%) needed to have the imipenem/cilastatin dose adjusted accordingly (Table 2).

\section{Indication}

Extensive clinical research marked the effectiveness of an initial monotherapy of imipenem/cilastatin in the treatment of moderate to severe infections, including septicemia, neutropenic fever, and intra-abdominal, lower respiratory tract, genitourinary, gynecological, skin and soft tissue, and bone and joint infections. ${ }^{16}$ The majority of the patients were diagnosed with urinary tract infections (27\%) typically due to an extended-spectrum $\beta$-lactamase-producing organism or sepsis of an unknown focus (22\%). The indication for the use of imipenem/cilastatin in the 100 patients is in Table 1. Nosocomial pneumonia was the indication in $19 \%$ and febrile neutropenia in $10 \%$ of the patients. Intra-abdominal, bone and joint, and diabetic foot infections were the least common, each accounting for only $2 \%$ of the patients.

The use of imipenem/cilastatin empirically was appropriate for the indication in $97.2 \%(n=69 / 71)$ of the

Table 2 Estimated baseline creatinine clearance for patients started on imipenem/cilastatin

\begin{tabular}{ll}
\hline $\begin{array}{l}\text { Estimated baseline creatinine } \\
\text { clearance }^{\mathbf{a}}(\mathrm{mL} / \mathrm{min})\end{array}$ & $\begin{array}{l}\text { Number of } \\
\text { patients }\end{array}$ \\
\hline$\geq 70$ & $49(49 \%)$ \\
$41-69$ & $1 \mathrm{I}(1 \mathrm{I})$ \\
$21-40$ & $24(24 \%)$ \\
$6-20$ & $16(16 \%)$ \\
\hline
\end{tabular}

Notes: aedian: $74.5 \mathrm{~mL} / \mathrm{min}$; interquartile range: $128 \mathrm{~mL} / \mathrm{min}$; sample size $\mathrm{n}=100$. cases. Two patients out of 71 (2.8\%) had been inappropriately prescribed imipenem/cilastatin empirically.

As for the assessment of imipenem/cilastatin indication postculture results, $11 \%$ of the patients were inappropriately started on and/or inappropriately continued imipenem/cilastatin after the culture results (Table 3 ).

Of the patients who were started on imipenem/cilastatin empirically, 78.8\% $(n=56 / 71)$ continued this treatment after the culture results. Seven of these patients $(12.5 \%, \mathrm{n}=7 / 56)$ were inappropriately kept on imipenem/cilastatin. Three patients $(5.3 \%, n=3 / 56)$ of those who were started on the antibiotic empirically did not have culture results taken (Table 3).

Twenty-nine percent of the patients were not started on imipenem/cilastatin empirically, four of whom (13.7\%, $n=4 / 29$ ) inappropriately received imipenem/cilastatin postculture results (Table 3).

\section{Dose}

Forty-nine percent of the patients had no (and did not develop) renal impairment $(\mathrm{CrCl}<70 \mathrm{~mL} / \mathrm{min})$ (Table 4). Fifty-one patients (51\%) either already had baseline renal impairment or had worsened kidney function while at the hospital $(\mathrm{CrCl}<70 \mathrm{~mL} / \mathrm{min})$. Out of the 49 patients who did not have renal impairment, only three $(6.1 \%)$ were dosed inappropriately. As for the rest of the patients (those with $\mathrm{CrCl}<70 \mathrm{~mL} / \mathrm{min}$ ), $58.8 \%$ ( $\mathrm{n}=30 / 51$ ) were not given an appropriate dose. In total (irrespective of the patients' renal function), 33 patients out of 100 were not dosed appropriately (Table 4).

\section{Monitoring of seizure occurrence}

Out of all the patients included in this study, only one patient developed a seizure. The patient presenting with nosocomial aspiration pneumonia was started on imipenem/cilastatin empirically after obtaining a sputum sample for culture. The culture result revealed the presence of Escherichia coli (extended-spectrum $\beta$-lactamase producer) in her sputum, and imipenem/cilastatin was continued based on her postculture results. She did not have renal impairment and her $\mathrm{CrCl}$ was stable $(>100$ $\mathrm{mL} / \mathrm{min}$ ) at all times. But it is important to note that she had a history of pre-existing CNS disease, evident by an old magnetic resonance image. Because of the seizure this patient developed, imipenem/cilastatin was discontinued and replaced by colistin (polymixin E) after consulting the infectious diseases team. 
Table 3 Appropriateness of imipenem/cilastatin use postculture

\begin{tabular}{|c|c|c|c|c|c|}
\hline \multirow{2}{*}{$\begin{array}{l}\text { Time of starting } \\
\text { Empirically }(7 \mathrm{I})\end{array}$} & \multicolumn{2}{|c|}{ Number of patients (n) } & \multirow{2}{*}{$\begin{array}{l}\text { Appropriate } \\
46(82.1 \%)\end{array}$} & \multirow{2}{*}{$\begin{array}{l}\text { Inappropriate } \\
7(12.5 \%)\end{array}$} & \multirow{2}{*}{$\begin{array}{l}\text { Culture not taken } \\
3(5.3 \%)\end{array}$} \\
\hline & Continued & $56(78.8 \%)$ & & & \\
\hline & Discontinued & $15(21.1 \%)$ & $15(100 \%)$ & 0 & 0 \\
\hline Nonempirically (29) & Started & 29 & $25(86.2 \%)$ & $4(13.7 \%)$ & 0 \\
\hline Total & & 100 & $86(86 \%)$ & II (II\%) & $3(3 \%)$ \\
\hline
\end{tabular}

\section{Discussion}

This is the first study conducted at this hospital that evaluates the appropriateness of imipenem/cilastatin prescription empirically, its dosage (and dosing adjustment whenever required) before and after culture results, and the rate of occurrence of seizures in the study patients.

Based on the results of this study, imipenem/cilastatin was appropriately used in $97 \%$ of the cases when it was prescribed empirically. A similar study that assessed the empirical use of imipenem/cilastatin conducted in a university hospital in Chile showed a lower rate of appropriateness as compared to our study. ${ }^{32}$ In that study, $58.1 \%(n=79 / 136)$ of empirical imipenem/cilastatin use was considered to be appropriate, while $11.8 \%$ was not appropriate $(n=16 / 136)$. The remaining patients either discontinued imipenem/cilastatin by physicians in charge prior to evaluation $(20.6 \% \mathrm{n}=28 / 136)$ or, unfortunately, died $(9.6 \% n=13 / 136) .{ }^{32}$ In another study, by Sakhaiyan et al, ${ }^{18}$ which also assessed the appropriateness of the use of imipenem/cilastatin empirically but in patients undergoing bone marrow transplantation at a single hospital and at a specific ward, 22 patients (35.9\%) needed dosage adjustments due to low weight or renal failure, but no action was taken at the time. Moreover, a study conducted in Iran by Shiva et $\mathrm{a}^{17}$ also addressed the same topic, and the authors came to the conclusion that the dosage of imipenem was inappropriate in $36 \%$ of patients, and the dosage adjustment (when needed) was either not done or done inappropriately in $64.3 \%(\mathrm{n}=9 / 14)$ of patients.

The high percentage of appropriateness in imipenem/ cilastatin prescription at our setting was due to the fact that the physicians were compliant with the current guidelines and,

Table 4 Appropriateness of the dose of imipenem/cilastatin

\begin{tabular}{llll}
\hline Renal status & $\begin{array}{l}\text { Number } \\
\text { of patients (n) }\end{array}$ & Appropriate & Inappropriate \\
\hline $\mathrm{CrCl} \geq 70 \mathrm{~mL} / \mathrm{min}$ & 49 & $46(93.9 \%)$ & $3(6.1 \%)$ \\
$\mathrm{CrCl}<70 \mathrm{~mL} / \mathrm{min}$ & $5 \mathrm{I}$ & $21(41.2 \%)$ & $30(58.8 \%)$ \\
Total & 100 & $67(67 \%)$ & $33(33 \%)$ \\
\hline
\end{tabular}

Abbreviation: $\mathrm{CrCl}$, creatinine clearance. most importantly, due to the implementation of a restriction policy at the hospital that allows the infectious diseases team to reject or authorize, within 24 hours, the use of imipenem/ cilastatin after an evaluation of its appropriateness for use.

When the use of imipenem/cilastatin based on postculture results was assessed, the degree of appropriateness decreased at our institution to $86 \%$, and that was after including patients who had been started on imipenem/cilastatin after the culture results were available. This was mainly due to the multiple chances of de-escalation that did not occur when possible. In addition, some patients were started on other antibiotics but kept on imipenem/cilastatin despite evidence that the microorganism identified turned out to be resistant to imipenem/cilastatin. In a few cases when imipenem/cilastatin was started after the culture results were out, four patients out of $29(13.8 \%)$ were inappropriately started on imipenem/ cilastatin. These patients either could have been placed on an antibiotic of a narrower spectrum $(n=1)$ or should not have been placed on imipenem/cilastatin in the first place, due to the resistance of the identified microorganism to the drug $(n=3)$. Thus, the appropriateness of use of imipenem/cilastatin with respect to indications at our hospital is relatively acceptable but could be further improved.

The dosing of imipenem/cilastatin assessed in this study was shown to be appropriate in only $67 \%$ of the cases. It is important to highlight the fact that the inappropriateness in the dosing regimen among the rest of the patients $(33 \%)$ was mainly evident in patients who already had a low $\mathrm{CrCl}(<70 \mathrm{~mL} / \mathrm{min})$ or those who had developed renal impairment while at the hospital. In total, three patients without baseline renal impairment and 30 patients with baseline renal impairment received an inappropriate dose of imipenem/cilastatin.

When there was no focus of infection, the dose of imipenem/ cilastatin prescribed was the average recommended dose in the references.

Guidelines are present for appropriate dosing of imipenem/ cilastatin in renal dysfunction, taking into account body weight, infecting pathogen, and $\mathrm{CrCl}^{33} \mathrm{It}$ is important to note that a considerable number of patients did not have their weight recorded 
on the electronic chart, thereby mandating the estimation of their weight.

The most widely used empirical dose of imipenem/ cilastatin was $500 \mathrm{mg}$ given intravenously four times daily, sometimes regardless of the patient's weight. This was one of the most documented causes of inappropriate initial dosing of imipenem/cilastatin. Another reason was that, in several cases, doses were not adjusted according to varying serum creatinine levels (whether improving or worsening). Guidelines emphasize the importance of periodic assessment of renal function and adjusting the dose accordingly. ${ }^{34}$ Frequent monitoring of the renal function of patients receiving imipenem/cilastatin is necessary. Patients with a $\mathrm{CrCl}$ of $<70 \mathrm{~mL} / \mathrm{min}$ require more intensive follow-up to adjust their dose of imipenem/cilastatin when needed.

This study also evaluated the propensity of seizures with the use of imipenem/cilastatin. Out of the 100 patients, only one female patient developed a seizure, which was myoclonic in nature and was associated with movement of the hands and deterioration of consciousness. She did not have renal impairment and her $\mathrm{CrCl}$ was stable $(>100 \mathrm{~mL} / \mathrm{min})$ at all times. Many clinical trials have assessed the rate of seizure occurrence in patients receiving imipenem/cilastatin, and patients who were at the highest risk of seizures were those who had a pre-existing CNS disease and/or a seizure history, those who were receiving higher than recommended doses, and those patients with renal insufficiency. The patient who developed a seizure was receiving $500 \mathrm{mg}$ intravenously four times daily, which was the appropriate dose for her. However, the patient had a history of pre-existing CNS disease, which increased her risk of developing a seizure while on imipenem/ cilastatin. This patient had several comorbidities alongside the nosocomial aspiration pneumonia, which made it difficult for us to establish a causal relationship with imipenem/ cilastatin. ${ }^{16}$ However, none of the other patients, including the 15 other patients (15\%) who also had pre-existing CNS disease and/or a history of seizure, developed any kind of seizure activity.

Appropriate imipenem/cilastatin prescribing, dosing, and monitoring proved to be of great impact on morbidity and mortality of hospitalized patients. In a study conducted by Thuong et al, it was found that only $75 \%$ of the empirical prescribing of antibiotics was later supported by culture results. ${ }^{35}$ Therefore, it is especially important that therapy be re-evaluated and, if necessary, modified 3-4 days after initiation of therapy and after culture results are available. The presence of a clinical pharmacist would help address the appropriateness in initial empirical prescription and then the re-evaluation of therapy after culture results are available, in a way that is compliant with the guidelines. Moreover, a study has shown that hospitals that have restricted carbapenem regulations use significantly fewer carbapenems $(P=0.04)$ and report significantly lower incidence rates of carbapenemresistant $P$. aeruginosa for up to 5 years $(P=0.01) .{ }^{36}$ Another study also concluded that carbapenem constraint could reduce the incidence of multidrug-resistant $A$. baumannii in ventilator-associated pneumonia $(P<0.05) .{ }^{37}$

There are several limitations to this study. First, it is an observational study; therefore, the cause-effect relationship between elements could not be ensured. Second, the observational period was relatively short, which led to a limited number of patients. Third, the study took place in one academic tertiary care center, which limits the ability to generalize the results. Fourth, keeping in mind that the Cockcroft-Gault method was used to provide a $\mathrm{CrCl}$ estimate and it requires information about age, sex, serum creatinine, and weight, some of the patients' body weights were estimated when they were not documented in the patients' records. Additionally, using the estimated $\mathrm{CrCl}$ equations for patients with acute kidney injury made the results difficult to interpret. Ideally, urine creatinine collection should be employed.

\section{Conclusion}

In general, this study evaluates prescribing patterns at our hospital and evaluates the compliance to national guidelines. The prescription of imipenem/cilastatin per indication at our hospital was mostly appropriate to what is recommended in the guidelines, although a few cases could have been managed better. Dosage adjustment, however, was not as appropriate, mainly in patients who did not have a stable $\mathrm{CrCl}$. The need for interventional actions by a clinical pharmacist on imipenem/cilastatin use is essential in the various units of the hospital. A clinical pharmacist may be able to help regulate the use of imipenem/cilastatin, ensure appropriate dosage adjustment when necessary, educate other health care professionals, improve patient outcomes, and decrease the length of hospital stay. ${ }^{38}$ In conclusion, the implementation of the restriction policy at our institution is the reason why the prescription of imipenem/cilastatin was close to ideal. However, to improve the dosage adjustment of imipenem/cilastatin, gathering of recorded data about inappropriate therapy should be provided to the physicians to be able to evaluate and optimize future drug therapies. In addition, further involvement of a clinical pharmacist in each hospital ward can lead to timely patient follow-up and the adjustment of imipenem/cilastatin dose when required. 


\section{Disclosure}

The authors report no conflicts of interest in this work.

\section{References}

1. Walker B, Barrett S, Polasky S, et al. Environment. Looming global scale failures and missing institutions. Science. 2009;325: 1345-1346.

2. Caldwell R, Lindberg D, editors. Understanding evolution: mutations are random [webpage on the Internet]. University of California Museum of Paleontology. Available from: http://evolution.berkeley.edu/ evolibrary/article/mutations_07. Accessed January 22, 2015.

3. Hawkey PM, Jones AM. The changing epidemiology of resistance. J Antimicrob Chemother. 2009;64(Suppl 1):i3-i10.

4. Pechère JC. Patients' interviews and misuse of antibiotics. Clin Infect Dis. 2001;33(Suppl 3):S170-S173.

5. Spellberg B, Blaser M, Guidos R, et al. Combating antimicrobial resistance: policy recommendations to save lives. Clin Infect Dis. 2011; 52(Suppl 5):S397-S428.

6. Zilberberg MD, Chen J, Mody SH, Ramsey AM, Shorr AF. Imipenem resistance of Pseudomonas in pneumonia: a systematic literature review. BMC Pulm Med. 2010;24:10.

7. National Nosocomial Infections Surveillance System. National Nosocomial Infections Surveillance (NNIS) System Report, data summary from January 1992 through June 2004, issued October 2004. Am J Infect Control. 2004;32:470.

8. Obritsch MD, Fish DN, MacLaren R, Jung R. National surveillance of antimicrobial resistance in Pseudomonas aeruginosa isolates obtained from intensive care unit patients from 1993 to 2002. Antimicrob Agents Chemother. 2004;48:4606-4610.

9. Araj GF, Zaatari GS. Antimicrobial Susceptibility Patterns of Bacterial Isolates at American University of Beirut Medical Center. Beyrouth: Wyeth Pharmaceuticals; 2001.

10. Slama TG. Gram-negative antibiotic resistance: there is a price to pay. Crit Care. 2008;12(Suppl 4):S4.

11. Centers for Disease Control and Prevention. Acinetobacter baumannii infections among patients at military medical facilities treating injured US service members, 2002-2004. MMWR Morb Mortal Wkly Rep. 2004;53(45):1063-1066.

12. Talbot GH, Bradley J, Edwards JE Jr, Gilbert D, Scheld M, Barlett JG. Antimicrobial Availability Task Force of the Infectious Diseases Society of America: bad bugs need drugs: an update on the development pipeline from the Antimicrobial Availability Task Force of the Infectious Diseases Society of America. Clin Infect Dis. 2006;42:657-668.

13. Lambert PA. Mechanisms of antibiotic resistance in Pseudomonas aeruginosa. J Roy Soc Med. 2002;95(Suppl 41):22-26.

14. Gaynes R, Edwards JR. National Nosocomial Infections Surveillance System: overview of nosocomial infections caused by gram-negative bacilli. Clin Infect Dis. 2005;41:848-854.

15. Clissold SP, Todd PA, Campoli-Richards DM. Imipenem/cilastatin. A review of its antibacterial activity, pharmacokinetic properties and therapeutic efficacy. Drugs. 1987;33:183-241.

16. Buckley MM, Brogden RN, Barradell LB, Goa KL. Imipenem/cilastatin: a reappraisal of its antibacterial activity, pharmacokinetic properties and therapeutic efficacy. Drugs. 1992;44:408-444.

17. Shiva A, Salehifar E, Amini M, et al. Drug utilization evaluation of imipenem in an educational hospital in Mazandaran Province. J Pharm Sci. 2014;20(1):12-17.

18. Sakhaiyan E, Hadjibabaie M, Gholami K, et al. Drug utilization evaluation of imipenem in patients undergoing bone marrow transplantation. Int J Hematol Oncol Stem Cell Res. 2009;3(2):10-13.

19. Brothrton TJ, Kelber RL. Seizure-like activity associated with imipenem. Clin Pharmacy. 1984;3:536-540.

20. Eng RH, Munsif AN, Yongco BG, et al. Seizure propensity with imipenem. Arch Intern Med. 1989;149:1881-1883.
21. Leo RJ, Ballow CH. Seizure activity associated with imipenem use: clinical case reports and review of the literature. Ann Pharmacother. 1991;25:351-354.

22. Calandra G, Lydick E, Carrigan J, et al. Factors predisposing to seizures in seriously ill, infected patients receiving antibiotics: experience with imipenem/cilastatin. Am J Med. 1988;84:911-918.

23. Gupta K, Hooton TM, Naber KG, et al. International clinical practice guidelines for the treatment of acute uncomplicated cystitis and pyelonephritis in women: a 2010 update by the Infectious Diseases Society of America and the European Society for Microbiology and Infectious Diseases. Clin Infect Dis. 2011;52(5):e103-e120.

24. Freifeld AG, Bow EJ, Sepkowitz KA, et al. Clinical practice guideline for the use of antimicrobial agents in neutropenic patients with cancer: 2010 update by the Infectious Diseases Society of America. Clin Infect Dis. 2011;52(4):e56-e93.

25. Stevens DL, Bisno AL, Chambers HF, et al. Practice guidelines for the diagnosis and management of skin and soft tissue infections: 2014 update by the Infectious Diseases Society of America. Clin Infect Dis. 2014;59(2):e10-e 52.

26. Lipsky BA, Berendt AR, Cornia PB, et al; Infectious Diseases Society of America. Executive summary: 2012 Infectious Diseases Society of America practice guideline for the diagnosis and treatment of diabetic foot infections. Clin Inect Dis. 2012;54:1679-1684.

27. Solomkin JS, Mazuski JE, Bradley JS, et al. Diagnosis and management of complicated intra-abdominal infection in adults and children: guidelines by the Surgical Infection Society and the Infectious Diseases Society of America. Clin Infect Dis. 2010;50:133-164.

28. Dellinger RP, Levy MM, Rhodes A, et al; Surviving Sepsis Campaign Guidelines Committee Including the Pediatric Subgroup. Surviving Sepsis Campaign: international guidelines for management of severe sepsis and septic shock: 2012. Crit Care Med. 2013;41(2): 580-637.

29. The Merck Manual. Imipenem and cilastatin [webpage on the Internet]. Lexi-Comp. Merck \& Co, Inc. 2013. Available from: http:// www.merckmanuals.com/professional/print/lexicomp/imipenem\%20 and\%20 cilastatin.html?qt=idarubicin\&alt=sh. Accessed January 22, 2015.

30. RxList. Primaxin IV (imipenem and cilastatin) for injection [webpage on the Internet]. Available from: http://www.rxlist.com/primaxin-ivdrug.htm. Accessed January 22, 2015.

31. McAuley D. Imipenem/cilastatin Primaxin ${ }^{\circledR}$ renal dosing [webpage on the Internet]. Available from: http://www.globalrph.com/imipenem_ renal.htm. Accessed January 22, 2015.

32. Fica CA, Luppi MM, Olivares CR, Brito FL, Zilleruelo LI, Muñoz CL. Imipenem-cilastatin use in a university hospital in Chile. Rev Chilena Infectol. 2006;23(4):307-315.

33. Imipenem [package insert]. Whitehouse Station, NJ: Merck \& Co; 2007. Available from: http://www.globalrph.com/imipenem_dilution. $\mathrm{htm}$. Accessed January 22, 2015.

34. Pastel DA. Imipenem-cilastatin sodium, a broad-spectrum carbapenem antibiotic combination. Clin Pharm. 1986;5:719-736.

35. Thuong M, Shortgen F, Zazempa V, et al. Appropriate use of restricted antimicrobial agents in hospitals: the importance of empirical therapy and assisted re-evaluation. J Antimicrob. Chemother. 2000;46(3): 501-508.

36. Pakyz AL, Oinonen M, Polk RE. Relationship of carbapenem restriction in 22 university teaching hospitals to carbapenem use and carbapenemresistant Pseudomonas aeruginosa. Antimicrob Agents Chemother. 2009;53(5):1983-1986.

37. Xue XS, Wang B, Deng LJ, Kang Y. [Carbapenem restriction reduce the incidence of multidrug-resistant Acinetobacter baumannii in ventilator associated pneumonia]. Chin Crit Care Med. 2009;21(4):234-236. Chinese

38. Kaboli P, Hoth A, McClimon B, et al. Clinical pharmacists and inpatient medical care: a systematic review. Arch Intern Med. 2006;166: 955-964. 
Infection and Drug Resistance

Dovepress

\section{Publish your work in this journal}

Infection and Drug Resistance is an international, peer-reviewed openaccess journal that focuses on the optimal treatment of infection (bacterial, fungal and viral) and the development and institution of preventive strategies to minimize the development and spread of resistance. The journal is specifically concerned with the epidemiology of antibiotic

resistance and the mechanisms of resistance development and diffusion in both hospitals and the community. The manuscript management system is completely online and includes a very quick and fair peerreview system, which is all easy to use. Visit http://www.dovepress.com/ testimonials.php to read real quotes from published authors.

Submit your manuscript here: http://www.dovepress.com/infection-and-drug-resistance-journal 